\title{
Research on a Specific Harmonic Elimination Technique for Step Wave of Cascade Type H Bridge Converters
}

\author{
Anqi Li \\ Department of Electrical Engineering, North China Electric Power University, Baoding 071000, \\ China
}

sumer951112@163.com

Keywords: selective harmonic elimination, the improved genetic algorithm, least squares optimization, 0-1 planning, numerical algorithm

Abstract. With the rapid development of power electronic technology, power electronic equipments with inverter devices as the main circuit structure are widely used, producing numerous harmonics and having a harmful effect on power grids and electricity equipments. This paper uses the improved genetic algorithm model and least square optimization model to calculate the switch angle when specific harmonic is eliminated and put forward the auxiliary control strategy to achieve power balance in each $\mathrm{H}$ bridge converter unit in a certain period.

\section{Restatement of the problem}

In occasions of high voltage or high power, more and more cascading multilevel converters are applied. Cascading multilevel converters are composed of several series of converter module units in order to realize the output of high voltage and multilevel. Its basic system structure is shown in figure 1. $\mathrm{H}$ bridge converter units with three level outputs are often used for the converter module, with the inductance L plays a role of filter.

In Selected Harmonic Elimination Pulse Width Modulation (SHEPWM)[1], a particular switch time is chosen to eliminate the selected low order harmonics when the desired output of fundamental wave voltage $v_{\mathrm{ac}}$ is met at the same time, thus improving the quality of output voltage waveform. Because the cascading multilevel converter output voltage $v_{\mathrm{ac}}$ is the superposition of $v_{\mathrm{aci}}$, output voltages of each $\mathrm{H}$ bridge converter unit, increasing the number of levels can make the output of the step wave closer to sine wave voltage to further reduce the harmonic content.

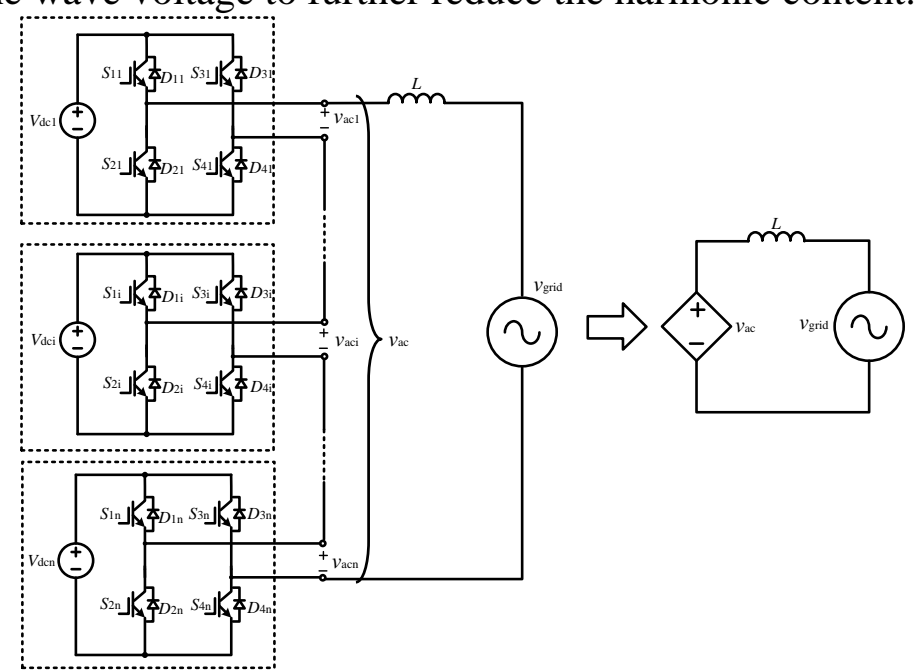

Figure 1: grid-connected system structure and the equivalent circuit based on cascading $\mathrm{H}$ bridges 


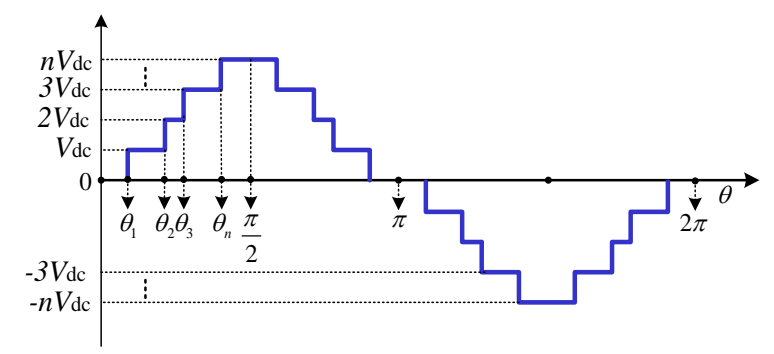

Figure 2: output voltage $v_{\text {ac }}$ wave form controlled by step wave SHEPWM

For the $\mathrm{i}^{\text {th }} \mathrm{H}$ bridge converter unit in cascading $\mathrm{H}$ bridge converter system, when $\left(S_{1 \mathrm{i}}, S_{3 \mathrm{i}}\right)$ or $\left(S_{1 \mathrm{i}}, S_{3 \mathrm{i}}\right)$ is open, 0 level is outputted, namely its output voltage $v_{\text {aci }}$ being 0 ; When $\left(S_{1 \mathrm{i}}, S_{4 \mathrm{i}}\right)$ is open, 1 level is outputted, namely its output voltage $v_{\text {aci }}$ being $V_{\text {dci }}$; When $\left(S_{2 \mathrm{i}}, S_{3 \mathrm{i}}\right)$ is open, -1 level is outputted, namely its output voltage $v_{\text {aci }}$ being $-V_{\text {dci }}$;

For cascading $\mathrm{H}$ bridge converter, the number of output level as a whole can be $(2 n+1)$. When all DC side independent voltage $V_{\mathrm{dci}}$ of $\mathrm{H}$ bridge converter unit being $V_{\mathrm{dc}}$, step voltage $v_{\mathrm{ac}}$ with output level number being $(2 n+1)$ can be outputted. As is shown in figure 2, output waveform of a single $\mathrm{H}$ bridge converter is always half wave odd symmetry and $1 / 4$ symmetry. Through Fourier series decomposition of the waveform, the amplitude of the $\mathrm{s}^{\text {th }}$ odd time harmonic of $v_{\text {ac }}$ can be expressed as expression (1):

$$
V_{s}=\frac{4 V_{d c}}{s \pi} \sum_{s=1,3,5, \cdots}^{\infty}\left[\cos \left(s \theta_{1}\right)+\cos \left(s \theta_{2}\right)+\cdots+\cos \left(s \theta_{n}\right)\right]
$$

In the expression: $0 \leq \theta_{1}<\theta_{2}<\cdots<\theta_{n} \leq \frac{\pi}{2}$ 。

For the cascading converter system with $\mathrm{n} \mathrm{H}$ bridge converter units, the number of selected harmonic elimination can be ( $n$ - 1 ) when meeting the expectations of fundamental voltage amplitude conditions. Then this paper tries to find out a set of $\theta_{\mathrm{i}}(i=1, \ldots, n)$ to make the fundamental component amplitude of the output voltage being V1m and low harmonic does not be contained (in symmetric three-phase system, harmonic with frequency being multiple of 3 is not considered).

Define modulation ratio amplitude $m=V_{1 \mathrm{~m}} /\left(n V_{\mathrm{dc}}\right)$, the nonlinear algebraic equations (2) of $\theta_{\mathrm{i}}$ $(i=1, \ldots, n)$ can be written according to the above constraints as follows:

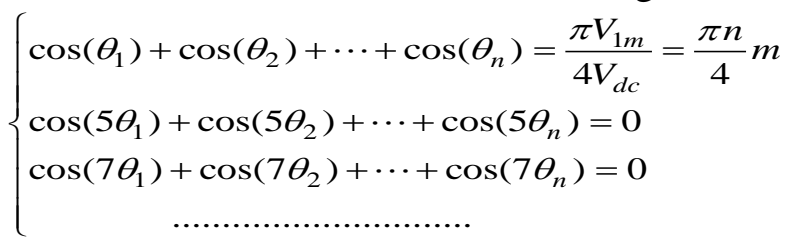

For a specific cascading modules number $n$, only when modulation ratio $m$ values within a certain range can the nonlinear system of equations has solutions. Solving equations require a set of initial value $\theta_{\mathrm{i}}{ }^{0} \quad(i=1, \ldots, n)$, which has a direct effect on convergence of equations solving.

At the same time, waveform quality of output voltage $v_{\text {ac }}$ based on step wave selected harmonic elimination technique can de described through the Total Harmonic Distortion (THD), as is shown in expression (3):

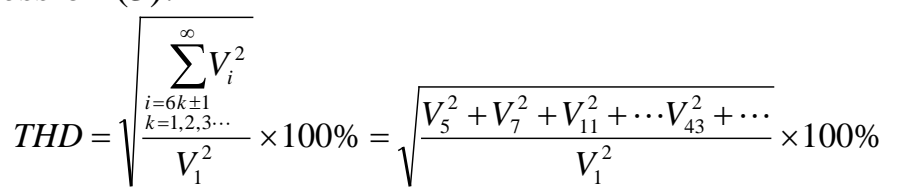

Mathematical model of selected harmonic elimination technique is a set of nonlinear equations, and the solution of the equations is the key factor that limits this technology in real-time application. 


\section{The establishment and solution of the model}

In order to achieve selected harmonic elimination, for each given set of cascading module number $n$ and eliminating harmonic frequency, there is a set of switch angle $\theta_{i}(i=1, \ldots, n)$ accordingly. In order to make $\theta_{i}(i=1, \ldots, n)$ has a solution, modulation ratio ought to have requirements of its range.

For getting the solution of the transcendental equation set of $S H E P W M$, researches mainly fall on two directions: numerical algorithm and heuristic algorithm. In terms of numerical algorithm, it relies heavily on the selection of initial value, and inappropriate initial values may directly cause the algorithm to have no convergence.

Compared with numerical algorithm, intelligent algorithm does not rely on the selection of initial values, therefore having good versatility and robustness. But it is likely to have defects like slow convergence rate and converging to local optimal solution.

\subsection{Genetic Algorithm}

Genetic Algorithm $(G A)$ is a kind of optimization search algorithm based on natural selection principle and natural genetic mechanism. It simulates the life evolution mechanism in the nature and achieves specific objectives optimization in the artificial system. The essence of genetic algorithm is to finally get the optimal solution or near optimal solution through group search technology and based on gradually evolution under the principle of survival of the fittest. It must follow the following steps: generating initial population, getting the fitness rate of each individual, choosing excellent individuals based on the principle of survival of the fittest, pairing each two excellent individuals, generating the next generation of group by random cross of chromosome gene and random mutation of some chromosome, making the group gradually evolve according to this method until meeting the termination conditions of evolution.

\subsubsection{Population initialization}

Because genetic algorithm has the characteristics of not depending on specific issues itself, the switch angle solution can be transformed to the encoding scheme (this paper is based on decimal encoding) that can be identified by the genetic algorithm. The encoding scheme randomly generates random number within the scope of $\left[0, \frac{\pi}{2}\right]$, making up the space solution matrix of $50 \times 3$, with the number of chromosomes being 50 as the size of the population. Each matrix chromosomes line refers to a randomly generated switch angle $\theta_{k}$.

\subsubsection{Selection}

According to the rules of survival of the fittest, individuals with higher fitness rate are more likely to be selected and survive. In order to measure the fitness rate of the individual, this paper constructs a fitness rate function to calculate the fitness rate of the individual. Then this paper adopts the method of roulette according to the fitness rate of individuals. The higher the fitness rate of the individual is, the greater the probability the individual can survive.

\subsubsection{Crossover}

Crossover operation refers to the operation of selecting two individuals at a certain probability and swapping the chromosomes after the intersection junction with the same position as the intersection junction. The purpose is to generate new hybrid individuals from the original parent individuals. For example, a population randomly generates two individuals $X_{1} 、 X_{2}$ on behalf of the switch angle $\alpha$. The two individuals carry on the cross with the second place being the intersection junction. Therefore, the first two positions of the chromosomes stay the same while positions afterwards become crossed. Each individual has three chromosomes.

\subsubsection{Mutation}

Mutation refers to the operation of choosing an individual at a certain probability and transforming its genes in random position, aiming to expand the diversity of solution. For example, the second position of the switch angle $X_{1}$ is used to do mutation operation, therefore numbers in the second position randomly generate again, while other positions stay unchanged. After operations like 
selection, crossover and mutation, the individual with the largest fitness rate is selected. If it meets the conditions of harmonic elimination, it is identified as the target angle solution; otherwise keep iteration until finding the optimal solution.

Although GA has many advantages in the optimization process, its biggest shortcoming falls on the fact that in the iterative process it is prone to become premature and make the algorithm converges to local optimal solution. The results show the fact that the quadratic sum of residuals of the equations is too big, causing the fact that using the result as the approximate solution is not accurate. This paper adopts an improved genetic algorithm to overcome the problems that standard $G A$ is prone to become premature and may prematurely converge to the second-best solution, improving it on the basis of the standard $G A$. The improvement lies in dynamically changing the probability of crossover and mutation. To some extent, it enables the algorithm to better adapt to the comprehensive searching to find the global optimal solution. This paper solves SHEPWM transcend equations corresponding to converters whose converter module unit has 3 levels output [2]and makes the switch angle converge to global optimal solution faster to find that scope of modulation ratio $m$ that enables $\theta_{i}(i=1, \ldots, n)$ a solution .

\subsection{The improved genetic algorithm}

The improved genetic algorithm can be divided into two modes: (1) a global search mode; (2) Local search mode. The characteristics of global search mode are high crossover probability and low mutation probability. It is used to reserve excellent parent genes as far as possible, at the same time in the global scope to make the algorithm evolve towards the objective function orderly in order to prevent it from being premature; while the characteristics of the local search mode are low crossover probability and high mutation probability. In this occasion, search is only done within the scope of target, focusing on finding the target optimal solution near the approximate optimal solution.

According to the characteristics of the SHEPWM equations, this paper focuses on the minimum value of the objective optimization function and defines fbest as the optimum solution in this generation, gbest as the optimal solution for past generations, $K$ as a constant that is no more than 1 . When $\frac{f b e s t}{\text { gbest }}<K$, the evolution is of high efficiency, and the algorithm uses the global search mode; otherwise adopts the model of local search.[3]

$i$ refers to the current evolution generations; $M$ refers to the total number of generations that have evolved; $P_{c 1}$ and $P_{m 1}$ respectively refers to the crossover probability and mutation probability in global search mode; $P_{c 2}$ and $P_{m 2}$ respectively refers to the crossover probability and mutation probability in local search mode.[4] In global search mode evolution direction will be more and more concentrated. Therefore the crossover probability and mutation probability can be dynamically adjusted according to the current evolution generation:

$$
\begin{aligned}
& P_{d}=P_{c 1 \text { max }}-\left(P_{c 1 \text { max }}-P_{c 1 \text { min }}\right) i / M \\
& P_{m 1}=P_{m 1 \text { max }}-\left(P_{m 1 \text { max }}-P_{m 1 \text { min }}\right) i / M
\end{aligned}
$$

In the expression, $P_{c 1 \text { max }}$ and $P_{c 1 \text { min }}$ respectively refers to the maximum and minimum of crossover probability; $P_{m 1 \max }$ and $P_{m 1 \min }$ respectively refers to the maximum and minimum of mutation probability. Local search pattern enjoys bigger variation rate and relatively random evolution direction. Therefore $P_{c 2} 、 P_{m 2}$ are set to be constants. According to considerable numbers of experiments, $P_{c 2} 、 P_{m 2}$ here were taken as 0.9 and 0.1 respectively, whose effect is great.

In this paper, SHEPWM equations of target optimization function can be transformed into:

$$
\left\{\begin{array}{c}
\cos \left(\theta_{1}\right)+\cos \left(\theta_{2}\right)+\ldots+\cos \left(\theta_{n}\right)-\frac{\pi n}{4} m=\varepsilon_{1} \\
\cos \left(5 \theta_{1}\right)+\cos \left(5 \theta_{2}\right)+\ldots+\cos \left(5 \theta_{n}\right)=\varepsilon_{2} \\
\cos \left(7 \theta_{1}\right)+\cos \left(7 \theta_{2}\right)+\ldots \cos \left(7 \theta_{n}\right)=\varepsilon_{3}
\end{array}\right.
$$


Total fitness rate function is: $\quad Y=\varepsilon_{1}^{2}+\varepsilon_{2}^{2}+\varepsilon_{3}^{2}$,

When $Y$ reaches its minimum, the equation set can be thought of having been solved.

\section{Acknowledgement}

This work was financially supported by Anqi Li.

\section{Reference}

[1] Chao Xu, Research on selected harmonic elimination technique equations quickly solve, I - II, 2007.

[2] Manyuan Ye, etc., 3 level neutral point clamped inverter selected harmonic elimination pulse width modulation technology based on feeding strategy genetic algorithm, High Voltage Technology, the $40^{\text {th }}$ volume, the $11^{\text {th }}$ phase : $3569-3576,2006$.

[3] Jianli Ding, etc., Combination of genetic algorithm and ant algorithm, Computer Research and Development, the $40^{\text {th }}$ volume, the $9^{\text {th }}$ phase: 1351-1351, 2003.

[4] Shoukui Si, Mathematical modeling algorithm and application, Beijing: National Defense and Industry Press, 2014. 